\title{
The "Radiation Continuity Checker", an Instrument for Monitoring Nuclear Disarmament Treaty Compliance
}

\author{
Accordingly the United States Gov- \\ A. Bernstein, B. A. Brunett, N. R. Hilton, J. C. Lund ${ }^{1}$, and J. M. Van Seyereont retains a non -exclusive, \\ Sandia National Laboratories, P.O. Box 969,Livermore, California 94551 \\ royalty-free license to publish or re. \\ produce the published form of this \\ contribution, or allow others to do so, \\ for United States Govexnmant pras- \\ poses.
} The submitted manuscalpt has boem anthored by a contractor of the United States Government under contract.

\section{Abstract}

We describe the design, construction and performance of an instrument designed to monitor compliance with future arms control treaties. By monitoring changes in the gamma-ray spectrum emitted by a stored nuclear weapon, our device is able to sense perturbations in the contents of a weapon storage container that would indicate treaty non-compliance. Our instrument (dubbed the Radiation Continuity Checker or RCC) is designed to detect significant perturbations in the gammaray spectra (indicative of tampering) while storing no classified information about the weapon, and having a negligible "false alarm rate".

In this paper we describe the technical details of two prototype instruments and describe the strategies we have adopted to perform signal processing in these instruments. Our first instrument prototype uses a scintillation spectrometer and a massive tungsten alloy collimator to reject the gamma-ray background. Our second prototype instrument makes use of an active collimation scheme employing a multiple detector Compton scatter approach to reject background radiation. The signal processing method we employ uses linear algorithms applied pulse by pulse. This eliminates the need for storage of pulse height spectra, which are in many cases classified.

\section{INTRODUCTION}

The successful implementation of future arms control treaties may be limited by the technical means used to verify various aspects of the nuclear weapon dismantlement process. A particularly vexing problem in treaty compliance is the problem of monitoring nuclear warheads that have been removed from a deployed position but are awaiting full disassembly. For instance, it has been estimated that it would take the U.S. five years to dismantle 1000 warheads. How could another party be assured that the US weapons were not tampered with or diverted during the lengthy period when the weapons are awaiting dismantlement and still in US possession? The instrument we are developing is intended to improve transparency during these periods. In particular, we are designing a device to monitor a nuclear weapon awaiting dismantlement and ensure - through a secure communication link and without revealing classified information - that the weapon remains unaltered ${ }^{2}$. The approach we have chosen to solve this problem is to use one or more small gamma-ray detectors mounted on a

1. Corresponding author; email: jlund@sandia.gov. nuclear weapon storage container. Built-in readout circuitry would automatically determine if any significant changes in the gamma emissions had occurred, and would communicate these changes to the outside world.

The RCC is designed to initialize only once. We envision that initialization would occur in the presence of representatives from both parties to a future treaty and would occur close to where the weapon was removed from its deployed position. After initialization, the RCC would remain securely attached to the weapon storage container and remain there until the weapon is verifiably destroyed. While attached to the storage container, the device could be polled at anytime via a secure radio-frequency link. The rate of polling would be dictated by security requirements, but we anticipate it would be at least once per day.

A major design difficulty is coping with changes in background radiation. We anticipate that the device will be initialized in a relatively low radiation background environment. After initialization, the weapon (and attached RCC) will be transported to a weapon storage facility to await dismantlement. The radiation background from other weapons in the storage facility is expected to be much higher than the background present at the initialization site. Indeed, for many possible storage configurations the background may be comparable in strength to the signal from the weapon to which the RCC is attached. Thus, a critical design requirement of the RCC is to greatly suppress radiation background while maintaining sensitivity to the weapon being monitored. If background suppression is not sufficient, "false alarms" could be induced with unacceptable frequency by changes in the background even if the weapon remained intact.Such false alarms would severely reduce the instrument's utility for arms control and treaty verification. Two approaches to background suppression were employed in our instruments: a passive approach employing a tungsten alloy collimator, and an active approach employing an "electronic collimator". The details of the two prototypes employing these different collimation approaches are discussed below.

2. For completeness, we note that our device may also be useful for monitoring stored pits in the potentially long waiting period following dismantlement and prior to destruction of the pit. This problem is in some respects easier to address because the signal strength from a stored pit is considerably greater than that of a stored weapon. 


\section{DISCLAIMER}

This report was prepared as an account of work sponsored by an agency of the United States Government. Neither the United States Government nor any agency thereof, nor any of their employees, make any warranty, express or implied, or assumes any legal liability or responsibility for the accuracy, completeness, or usefulness of any information, apparatus, product, or process disclosed, or represents that its use would not infringe privately owned rights. Reference herein to any specific commercial product, process, or service by trade name, trademark, manufacturer, or otherwise does not necessarily constitute or imply its endorsement, recommendation, or favoring by the United States Government or any agency thereof. The views and opinions of authors expressed herein do not necessarily state or reflect those of the United States Government or any agency thereof. 


\section{DISCLAIMER}

\section{Portions of this document may be illegible in electronic image products. Images are produced from the best available original document.}




\section{PASSIVE COLlimatoR PROTOTYPE}

\section{A. Design Overview}

Our first prototype RCC instrument was designed to use gamma-ray pulse height information to detect changes in the emissions from a weapon storage container. We used a scintillation spectrometer inside a tungsten alloy background shield (collimator) for the detector assembly. A standard pulse height spectroscopy chain of analog electronics was used to read out the detector, and an ADC controlled by a microcontroller was used to recover gamma-ray spectroscopic information from the detector. The microcontroller also performed the signal processing necessary to initialize and detect changes in the contents of the container. Photographs of the completed RCC assembly are shown in Figure 1 and Figure 2.

\section{B. Detector Design}

The detector used in our first prototype consisted of a $1 \mathrm{in}$. $\mathrm{NaI}(\mathrm{Tl})$ scintillator (Bicron 1R1 or Rexon 1x1) attached to a small photomultiplier tube (Hamamatsu R4700U). The active diameter of the PMT was only about $9 \mathrm{~mm}$, so only a fraction of the light from the scintillator was read out. Good energy resolution was not a requirement in this instrument, but small physical size was; thus we chose the small PMT because it enabled construction of one of the smallest detector assemblies possible using only commercially available components.

The scintillator/PMT assembly was mounted inside a tungsten alloy collimator with the PMT pointing toward the collimator opening. A photograph of one of the tungsten collimators is shown in Figure 3. The thickness of the collimator was determined by estimating the anticipated radiation environment and computing the transport of this estimated radiation through the collimator. The criteria used to determine collimator thickness was the attenuation required to suppress this background intensity such that it was no greater than $1 \%$ of the intensity of the gamma-ray emissions from the weapon being monitored. The shape of the collimator was arrived at by constraining the field of view of the detector to include the central axis of the storage container but to exclude radiation originating from outside the container. The total collimator mass was $12 \mathrm{~kg}$.

\section{Electronic Readout}

The electronics used to readout the detector in the passive RCC prototype consisted of the usual analog pulse processing components used in pulse height spectroscopy. The analog "front end" consisted of a charge sensitive preamplifier, a shaping amplifier and a peak sample and hold. The analog processing components were implemented on a single ASIC fabricated using Honeywell's ALB1A bipolar process. Additional details of the analog ASIC we used in our first prototype are given in [1].

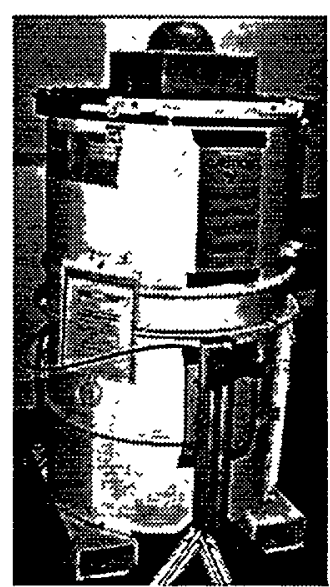

OSTI
Figure 1: Photograph of the RCC passive collimator prototype on top of a mock warhead storage container. The domed box at the top of the storage container contains the entire RCC unit (detector, collimator, and electronics) housed in a tamper-resistant fiberglass package. The passive tungsten alloy collimator is aimed down towards the warhead location in the container.

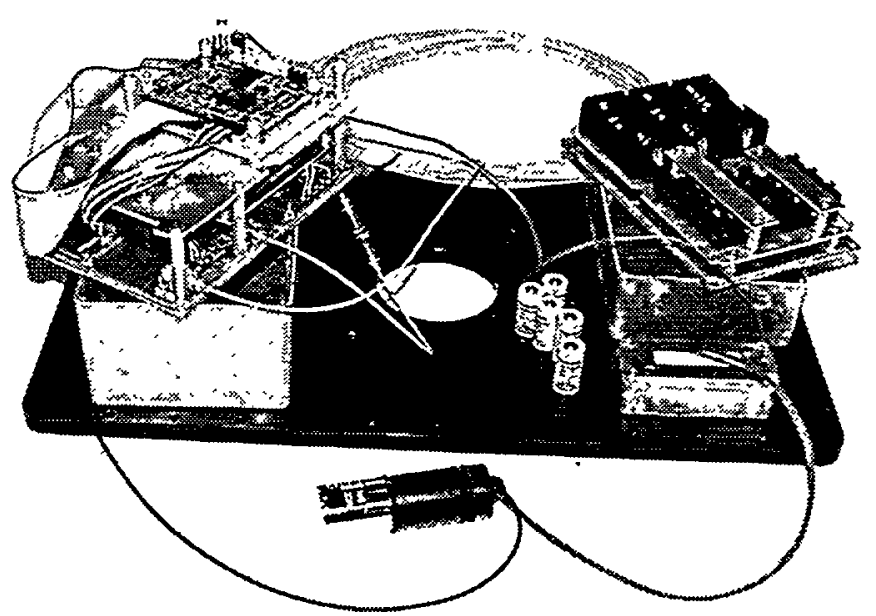

Figure 2: Photograph of the partially disassembled internals of the RCC passive collimator prototype. On the left is the analog and digital electronics package (signal processing, ADC, microcontroller, and RF transceiver electronics), on the right are the power supply batteries and high voltage power supply, and at the bottom center is the 1" Nal/ PMT detector removed from it's collimator.

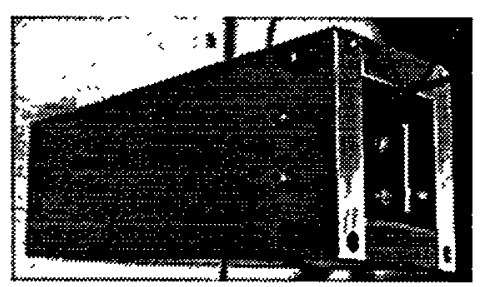

Figure 3: Photograph of a tungsten alloy collimator used in the RCC passive collimator prototype. The high voltage and signal leads to the scintillator/PMT detector can be seen on the right of the picture exiting from the bottom of the collimator. 
The peak sample and hold output of the ASIC was sampled with an Maxim MAX152 8-bit ADC connected to a Zworld microcontroller board. The microcontroller board also performed signal processing functions and communication with the radio frequency communications unit. After assembling and debugging the electronics unit it was determined that the microcontroller ADC combination could not operate fast enough to perform signal processing in near real-time. Instead, the microcontroller was programmed to act like a pulse height spectrometer and pulse height spectra were acquired and later operated on by the signal processing programs.

\section{Signal Processing}

The signal processing used in the first prototype unit was designed to implement a sequence of steps:

1. initialize (acquire a radiation signature),

2. store the signature (or a quantity derived from it),

3. acquire another radiation signature (on demand at some later time), and

4. compare the signature acquired later with the initial signature and decide if a significant perturbation in the contents of the weapon storage container had occurred.

Our initial signal processing experiments with the passive collimator prototype made use of a correlation algorithm to perform the similarity check between pulse height data acquired initially and in subsequent samples. The correlation algorithm was very effective in sensing small changes in the pulse height spectrum. In particular, we performed the discrete correlation of the N-element initial pulse height vector PHO, with a pulse height spectrum acquired later of the same length PH1. The resulting correlation vector $\mathbf{C}$ was then summed to produce a scalar value, $S 1$. $S I$ may be thought of as a "similarity scalar". When $S I$ is large the spectrum acquired at a later time is very similar to the spectrum acquired initially. Conversely, when $S 1$ is small, the most recently acquired pulse height spectrum deviates strongly from the initial spectrum, and it is likely that a major change in the radiation signature has occurred (indicating an alarm condition). $S 1$ is therefore a measure of the similarity of the subsequent spectrum to the initial one.

Although the correlation algorithm was an excellent indicator of changes in the radiation emanating from the weapon storage container, the correlation algorithm requires the storage of pulse height spectra, and pulse height histogram storage would cause classification and security problems. Another algorithm that we used with the passive collimator prototype showed similar performance to the correlation algorithm without the need for direct storage of spectral information.

The second algorithm we used in the passive collimator prototype is akin to the basic dot product of linear algebra. Assume that the pulse height spectrum acquired from the scintillation detector is represented by the vector $\mathbf{H}$, where the elements of $\boldsymbol{H}$ represent counts and the indices of $\boldsymbol{H}$ represent the pulse amplitude. Further assume that there exists a vector W, which may be thought of as a weighting vector. If we take the dot product of $\mathbf{H}$ and $\mathbf{W}$ we obtain a scalar value $S 2$, which may be thought of as measure of the similarity of the pulse height spectrum $\mathbf{H}$ to the generic weighting vector $W$. Note that the weighting vector, which must be stored in memory, does not have to contain any classified information. In our trials we obtained good performance using a simple weighting vector which consisted of a set of coefficients that increased in a linear fashion.

\section{ACTTVE COLlIMATOR PROTOTYPE}

\section{A. Design Overview}

Although good sensitivity to changes in the contents of a weapon storage container were obtained with our passive collimator prototype, the mass of the passive collimator seriously degraded the utility of the instrument for widespread field use. We decided that it might be feasible to replace the passive collimator with a lighter-weight "electronic collimator" based on Compton scattering of gamma-rays amongst multiple detectors. We chose a simple three detector design, utilizing commercially available $\mathrm{NaI}(\mathrm{Tl})$ scintillator elements. As with the passive collimator prototype, our active collimator employed a local computer to provide flexibility in evaluating signal processing algorithms. A photograph of the active collimator prototype is shown in Figure 4.

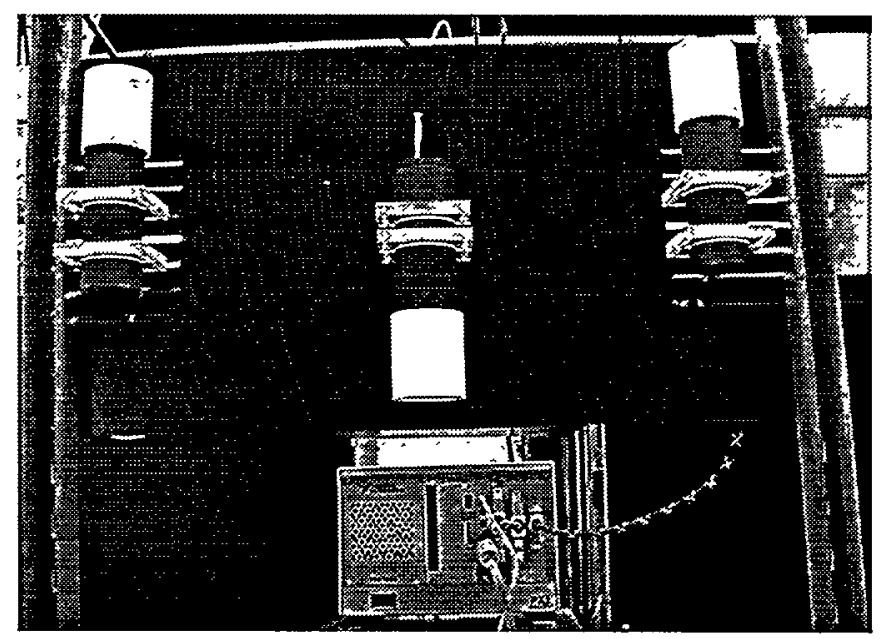

Figure 4: Photograph of the RCC active collimator prototype. The lower scatter and two upper calorimeter Nal/PMT detectors are prominent in the top center of the picture. The PXI controller system is visible in the bottom center. Not seen on the rear of the detector mounting plate is the box containing the analog signal processing and coincidence electronics.

\section{B. Detector Design}

Three detectors were used in the active collimator prototype, arranged in a triangular geometry, as shown in Figure 5. The arrangement can be thought of as two independent pairs of coincidence detectors. In the normal deployment mode the two rear detectors are set at an angle of 40 degrees relative to a vec- 
tor perpendicular to the surface of the weapon storage container through the center of the "front" detector. The detector electronics system was designed to measure photons in the $400 \mathrm{keV}+/-50 \mathrm{keV}$ region from the decay of ${ }^{239} \mathrm{Pu}$. At $400 \mathrm{keV}$ a photon scattering 40 degrees from the front detector to one of the rear detectors would deposit $62 \mathrm{keV}$ in the front detector and $338 \mathrm{keV}$ in one of the rear detectors; this partitioning of the total photon energy insures that all signals are well above the lower level discriminator levels.

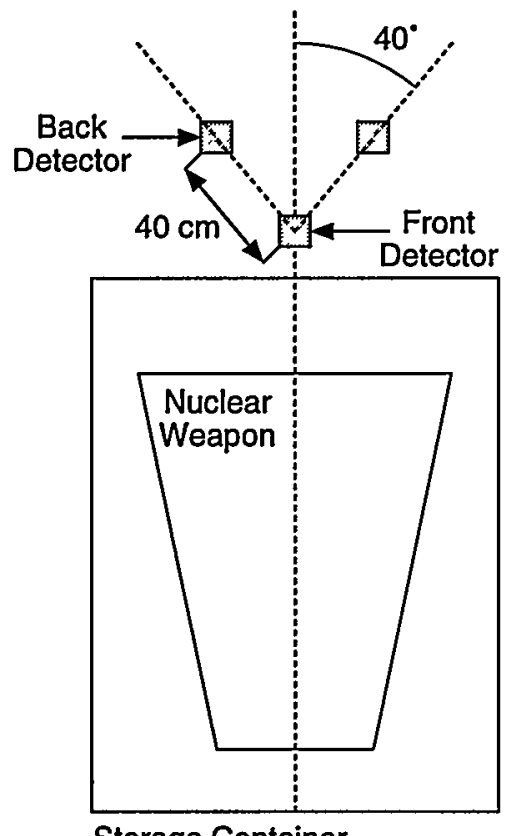

Storage Container

Figure 5: Diagram of the geometry used for the RCC active collimator prototype. The gamma-rays from the weapon in the storage container that Compton scatter from the front detector into either of the rear detectors are those of interest. The scatter angle used was chosen as the best tradeoff between sensitivity to changes in the weapon, lowest sensitivity to background outside the storage container, and deposited energy in the front detector.

There are two drawbacks of the 40 degree detector angle. The first is that the backprojected Compton cone sweeps out a substantial volume outside of the storage container being monitored, as shown diagrammatically in Figure 6. The second is that the data rate drops by a factor of about two relative to the rate for shallow-angle Compton scattering (i.e. scatter angle near zero degrees). Conversely, if a smaller angle is used between the front and rear detectors- such that the back-projected Compton cone lies completely inside the container - too little energy is deposited in the front detector to reliably distinguish the scattering event. The 40 degree angle between the front and rear detectors is a compromise between favorable energy partitioning amongst the detectors on the one hand, and background rejection and overall data rate on the other. Although the background will not be effectively rejected with a single detector pair, we believe that signal processing algo- rithms can be developed that use both pairs of detectors to increase background rejection.

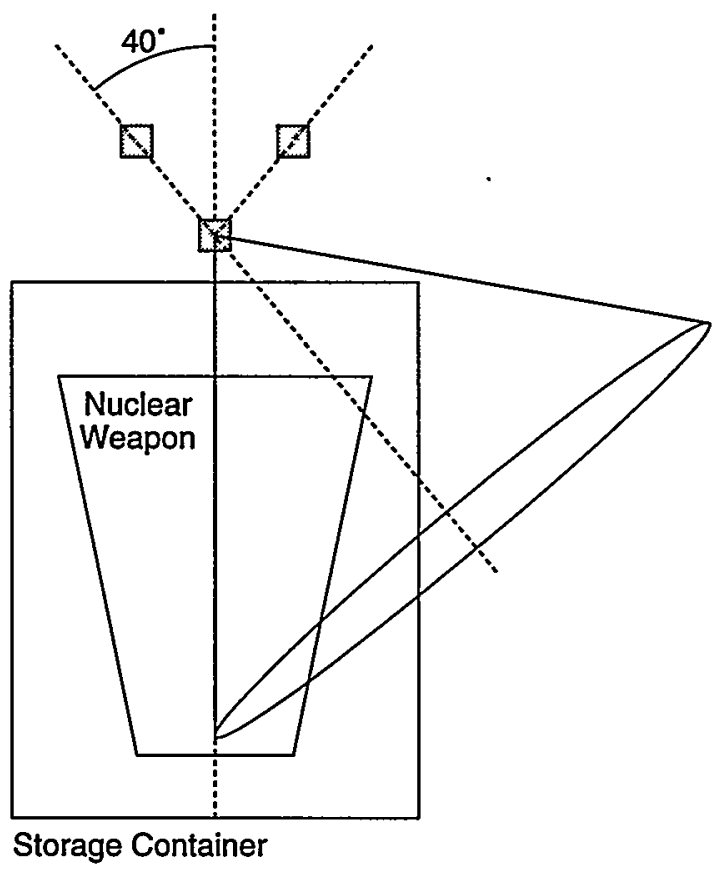

Figure 6: Diagram of the view obtained from the backprojection cone for a scatter event between the first pair of detectors. A large fraction of the view is outside the storage container, and is thus sensitive to the external background. This undesirable situation arises because of the need to have a minimum scatter angle to deposit sufficient energy in the front detector. The problem is resolved throught the use of both pairs of scatter detectors.

\section{Electronic Readout}

The electronic readout system we used in our active collimator prototype consisted of three channels of pulse height spectroscopy, coincident logic, and a computer acting as a multi-parameter analyzer. The analog front-end (preamplifier, shaper, peak sample and hold, and coincidence logic) were implemented in discrete components (no ASIC's), thus anticipating and avoiding future security issues. Pulse height acquisition was performed with a National Instruments PXI-6025E data acquisition board inside a PXI crate. The data acquisition board used a single 12-bit ADC and an analog multiplexor to sequentially read the three channels. Data acquisitions and signal processing were controlled by a Pentium-based PXI bus controller card, running Microsoft Windows 98 and National Instruments LabVIEW 5.0. An example of data acquired with the active collimator prototype is shown in Figure 7.

\section{Signal Processing}

As of this writing, software to perform the signal processing in the electronic collimator prototype is still under development. However, we anticipate using a dot product algorithm, similar to that used in the passive collimator prototype. 


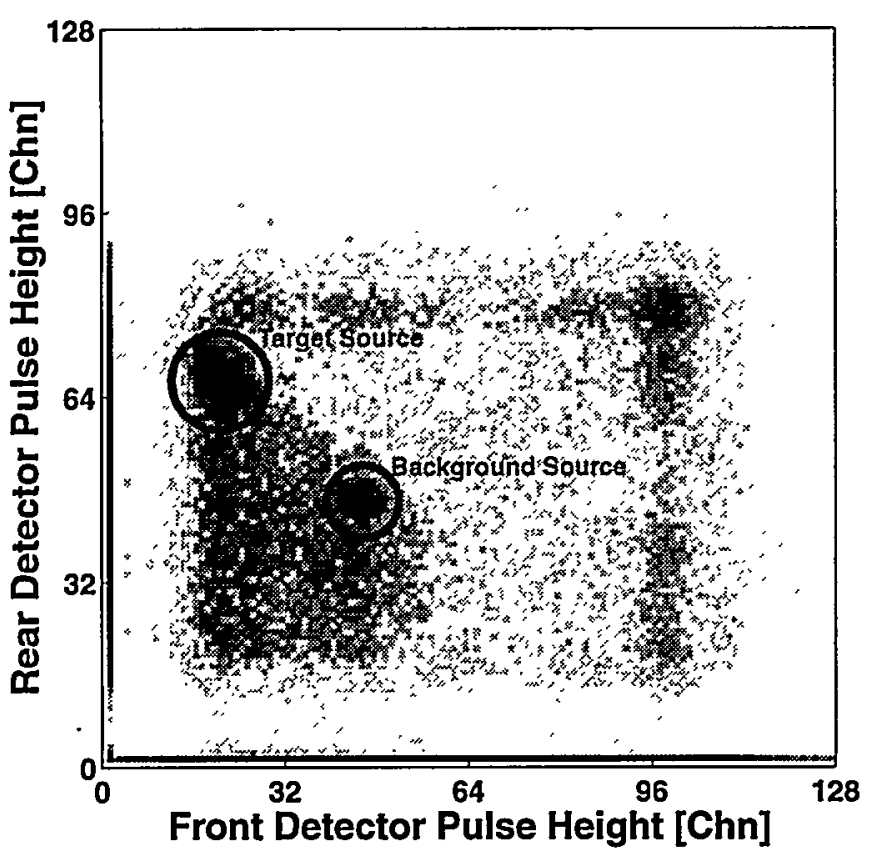

Figure 7: Example multi-parameter data obtained with the PXI-cratebased RCC active collimation prototype. The horizontal axis is the pulse height in the front detector, while the vertical axis is the pulse height in one of the rear detectors. This dataset was obtained for a

${ }^{133} \mathrm{Ba}$ source placed on the system centerline, and a separate ${ }^{133} \mathrm{Ba}$ source placed effectively at the container edge, although no container was present. The features resulting from the "sample" and "background" are indicated on the plot.

\section{SIGNAL PROCESSING ISSUES}

Both of our prototype RCC units constructed so far employed local computers to perform the signal processing. In the future, we would like to develop units that do not use microprocessors. A microprocessor-free unit is highly desirable from a security point of view because its operation can be more easily reverse engineered to insure that it is performing as intended. In addition, it is very desirable if the unit does not store any acquired pulse height information. Stored pulse height information may reveal classified information about the weapon being monitored, and any unit containing classified information would be subject to awkward handling procedures to ensure the integrity and security of the stored classified data.

The absence of a microprocessor and pulse height memory seem, initially, to be severe limitations on the construction of an RCC unit. However, after studying the various types of signal processing options available, we believe there may still be a large class of suitable algorithms. In particular, any linear algorithm may be deployed without storing the pulse height spectrum in memory. After all, linearity is just the condition that the algorithm will produce the same result irrespective of whether it operates on a complete pulse height histogram or on one pulse at a time. Thus, any linear algorithm can be effectively applied on a pulse by pulse basis with no need to store a histogram. For example, consider the example of the dot product algorithm we discussed earlier. This algorithm produces the same scalar result if a running sum is kept (by multiplying each pulse by an appropriate coefficient and summing) as it does if the dot product is used on the stored histogram.

What is less clear is how to best couple Compton scatter data from multiple detectors using a linear algorithm. Conventional backprojection algorithms often employ a nonlinear step (thresholding) to distinguish localized information above background ([2] and [3]) and we are not yet certain if a purely linear algorithm can be employed effectively. Of course, even if an algorithm is theoretically possible to use, implementing it without a microprocessor and using simple hardware may be a challenge.

\section{CONCLUSIONS}

We have built and tested two instruments to monitor stored nuclear weapons awaiting dismantlement. The use of gammaray spectroscopy to look for changes in the contents of a weapon storage container is, in principle, a straightforward problem to solve. However, the constraints imposed by large changes in background radiation and security restrictions make the design of an effective instrument very challenging. Our research to date indicates that Compton-based electronic collimation is a very promising approach. However, it remains to be seen whether this method can be rendered effectively without using a microprocessor or a stored pulse height spectrum.

\section{ACKNOWLEDGMENTS}

We gratefully acknowledge financial support from the Sandia National Laboratories WMTP project. We would also like to acknowledge Kurt Wessendorf and Jim Clements from Sandia/Albuquerque for assisting in the design and construction of the electronic readout used in both prototype instruments. Finally we would like thank Gary Hux and Kit Schmitz for assistance in mechanical design and construction.

\section{REFERENCES}

[1] K. O. Wessendorf, J. C. Lund, B. A. Brunett, G. R. Laguna, and J. W. Clements, "Very Low-Power Consumption Ana$\log$ Pulse Processing ASIC for Semiconductor Radiation Detectors", 1998 IEEE Nuclear Science Symposium and Medical Imaging Conference Conference Record, paper number N20-29 (1999).

[2] R. W. Todd, D. B. Everett, and J. M. Nightingale, "A Proposed Gamma Camera", Nature, Vol. 251, pp. 132-134 (1974).

[3] J. E. McKisson, P. S. Haskins, D. P. Henderson, Jr., K. C. Neelands, C. K. Wang, and T. Evans, "3-D Imaging of Complex Source Fields with a Compton Camera Imager", IEEE Trans. Nucl. Sci., Vol. 44, pp. 916-921 (1997). 